\title{
Decentralized Adaptive Double Integral Sliding Mode Controller for Multi-Area Power Systems
}

\author{
Bui Le Ngoc Minh, ${ }^{1}$ Van Van Huynh $\mathbb{D D}^{2},{ }^{2}$ Tam Minh Nguyen, ${ }^{1}$ and Yao Wen Tsai $\mathbb{D}^{3}$ \\ ${ }^{1}$ Faculty of Electrical and Electronics Engineering, Ho Chi Minh City University of Technology and Education, \\ Ho Chi Minh City, Vietnam \\ ${ }^{2}$ Modeling Evolutionary Algorithms Simulation and Artificial Intelligence, Faculty of Electrical \& Electronics Engineering, \\ Ton Duc Thang University, Ho Chi Minh City, Vietnam \\ ${ }^{3}$ Department of Mechanical and Automation Engineering, Da-Yeh University, Changhua 51591, Taiwan
}

Correspondence should be addressed to Van Van Huynh; huynhvanvan@tdtu.edu.vn

Received 12 February 2018; Revised 31 August 2018; Accepted 3 September 2018; Published 8 October 2018

Academic Editor: Adrian Chmielewski

Copyright (C) 2018 Bui Le Ngoc Minh et al. This is an open access article distributed under the Creative Commons Attribution License, which permits unrestricted use, distribution, and reproduction in any medium, provided the original work is properly cited.

\begin{abstract}
Most of the existing results for load frequency control of multi-area interconnected power systems can only be obtained when the norm of the aggregated uncertainties is bounded by a positive constant. This condition is difficult to achieve in real multi-area interconnected power systems. In this paper, a new load frequency control (LFC) for multi-area interconnected power systems is developed based on a decentralised adaptive double integral sliding mode control technique where the above limitation is eliminated. First, an adaptive gain tuning law is adopted to estimate the unknown upper bound of the aggregated uncertainties. Second, a double integral sliding surface based adaptive sliding mode controller is proposed to improve the transient performance of the closed loop system. Simulation results show that the proposed control law results in shortening the frequency's transient response, avoiding the overshoot, rejecting disturbance better, maintaining required control quality in the wider operating range, and being more robust to uncertainties as compared to some existing control methods.
\end{abstract}

\section{Introduction}

Load-frequency control (LFC) plays an important role in the operation of interconnected power systems to regulate the frequency and the tie line interchanges among different control areas [1]. There are many different control methods, which have been proposed in designing load frequency controllers with better performance to maintain the frequency and to keep tie line power flows within prespecified values during the last two decades [1-18]. The most conventional decentralized control methods for LFC are proportionalintegral control [1], proportional-double integral control [2], and PID control [3, 4]. PI control has the advantage of a simple controller structure. But it can yield a long settling time and a large overshoot in transient response [4]. PID controller is an effective LFC when the system is operating in the vicinity of the nominal operating point. However, the operating points could deviate from their nominal values significantly due to wearing out of components, the variations of power consumptions, the variations in synchronizing power coefficients, system uncertainties, and the change of the number of power plants in different control areas. The performance of a PI or PID controller would be significantly degraded due to a large deviation of operating points from their nominal values. In order to overcome the limitations of PI or PID control approaches, many advanced control methods such as optimal control [58], the intelligent proportional-integral LFC using genetic algorithms [9-11], internal model control [12], and model predictive control [13] are developed to maintain power quality in a wide range of operation. Recently, application of fuzzy logic controller to LFC has also been presented [1417]. To achieve a good fuzzy logic controller, the fuzzy rules must be correctly formulated, so the designer must have a thorough knowledge of the problem in formulating the rules. This is one of the most important features determining the 
quality of this type of control. In addition, several researchers have used state observer and disturbance observer-based controller to deal with the LFC problem. Liu et al. [18] proposed a full-order generalized state observer for load frequency control of multi-area interconnected power system. In [19], an output feedback controller based on universal finite-time observer is designed to regulate the frequency of the hydraulic turbine system. In principle, an exact system model has to be known to design such state observer and disturbance observers, which hardly holds in practice due to uncertain parameters (e.g., variations in synchronizing power coefficients, inertia, and damping parameters) in the system.

Among these presented control methods, sliding mode control (SMC) is recognized as one of the most efficient tools due to its fast response and strong robustness with respect to uncertainties and external disturbances [20]. Recently, there has been an increasing research interest in the sliding mode based load frequency control for power systems with matched and unmatched uncertainties [20-24]. In [20], a full-state sliding mode controller was developed for load frequency control of power systems. In order to improve system dynamic performance in reaching intervals, the decentralized sliding mode controller based on integral switching surface was designed for multi-area interconnected power systems [21]. The scheme of sliding mode control by model order reduction for the LFC problem of micro hydro power plants was addressed in [22]. In [23], the neural-network-based integral sliding mode controller was employed to achieve the LFC problem. In [24], a load frequency control strategy based on sliding mode control theory and disturbance observer was proposed for the single area power system. The above works obtained important results related to the load frequency control of interconnected power systems using SMC theory. As a result, the stability of interconnected power systems was assured under certain conditions. However, the traditional SMC method given in $[21,23,24]$ is based on integral sliding surface which may take long settling time and high overshoot.

Motivated by the aforementioned analysis, this paper proposes a new load frequency control for a more general structure of multi-area interconnected power systems based on the decentralised adaptive double integral sliding mode control technique. The main contributions of this paper are as follows.

(i) A double integral sliding surface based adaptive sliding mode controller is proposed to alleviate the steady-state errors and improve the transient performance of the closed loop system.

(ii) An adaptive gain tuning law is adopted in the proposed double integral sliding mode controller to estimate the unknown upper bound of the system uncertainty.

(iii) The two major limitations in $[21,23]$ (the lumped uncertainties are bounded by a positive constant and steady-state errors will be existent in the frequency deviation) are both eliminated.

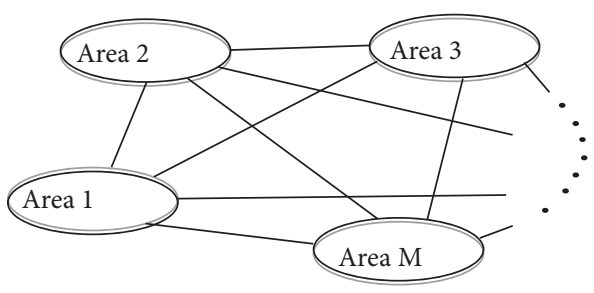

FIGURE 1: Multi-area interconnected power system.

(iv) The proposed control law results in shortening the frequency's transient response, avoiding the overshoot, rejecting disturbance better, maintaining required control quality in the wider operating range, and being more robust to uncertainties as compared to some existing control methods.

\section{A Multi-Area Interconnected Power System Model}

Without loss of generality, a multi-area interconnected power system same as $[21,23,24]$ is considered in this paper; see Figures 1 and 2. Although a power system is nonlinear and dynamic, the use of the linearized model is permissible in the load-frequency control problem because only small changes in load are expected during its normal operation [21, 23, 24].

The dynamic equations of the $i$ th area of a multi-area power system are as follows:

$$
\begin{aligned}
& \Delta \dot{f}_{i}(t) \\
& =-\frac{1}{T_{P_{i}}} \Delta f_{i}(t)+\frac{K_{P_{i}}}{T_{P_{i}}} \Delta P_{g_{i}}(t)-\frac{K_{P_{i}}}{T_{P_{i}}} \Delta P_{d_{i}}(t) \\
& \quad-\frac{K_{P_{i}}}{2 \pi T_{P_{i}}} \sum_{j=1}^{N} K_{s_{i j}}\left\{\Delta \delta_{i}(t)-\Delta \delta_{j}(t)\right\} \\
& \Delta \dot{P}_{g_{i}}(t)=-\frac{1}{T_{T_{i}}} \Delta P_{g_{i}}(t)+\frac{1}{T_{T_{i}}} \Delta X_{g_{i}}(t) \\
& \Delta \dot{X}_{g_{i}}(t)=\frac{-\Delta f_{i}(t)}{R_{i} T_{G_{i}}}-\frac{\Delta X_{g_{i}}(t)}{T_{G_{i}}}-\frac{\Delta E_{i}(t)}{T_{G_{i}}}+\frac{1}{T_{G_{i}}} u_{i}(t)
\end{aligned}
$$

$\Delta \dot{E}_{i}(t)$

$$
=K_{E_{i}}\left[K_{B_{i}} \Delta f_{i}(t)+\frac{1}{2 \pi} \sum_{\substack{j=1 \\ j \neq i}}^{N} K_{s_{i j}}\left\{\Delta \delta_{i}(t)-\Delta \delta_{j}(t)\right\}\right]
$$

$$
\Delta \dot{\delta}_{i}(t)=2 \pi \Delta f_{i}(t)
$$

where $i=1,2, \ldots, N$ and $N$ is the number of areas. The matrix form of the dynamic equations (1)-(5) can be written as

$$
\dot{x}_{i}(t)=\bar{A}_{i} x_{i}(t)+\bar{B}_{i} u_{i}(t)+\sum_{\substack{j=1 \\ j \neq i}}^{N} \bar{H}_{i j} x_{j}(t)+\bar{F}_{i} \Delta P_{d i}(t)
$$




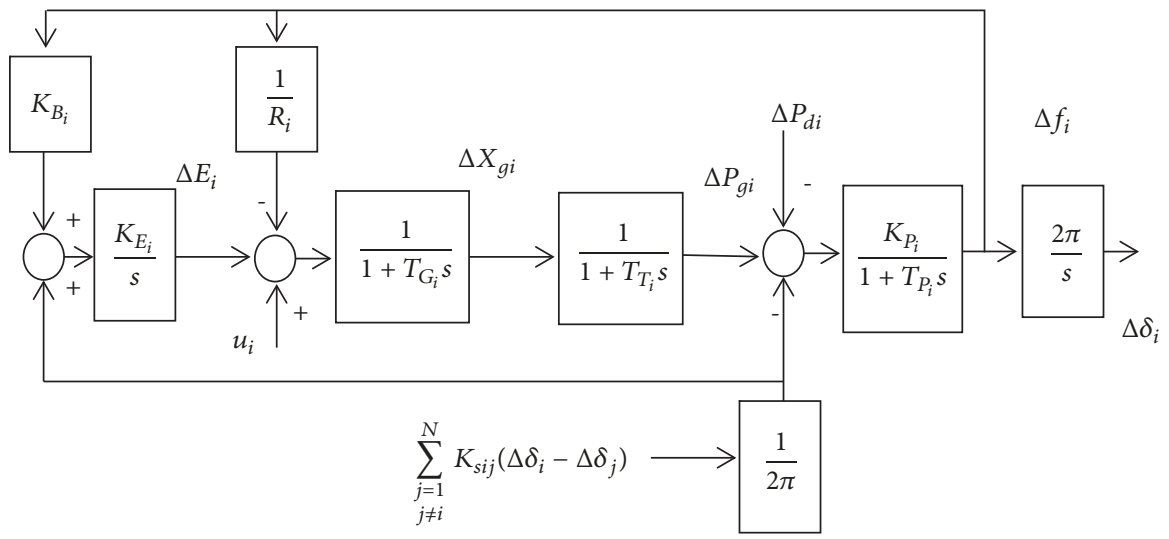

FIGURE 2: Block diagram of the $i$ th area of a multi-area power system.

where

$$
\begin{aligned}
& \bar{A}_{i}=\left[\begin{array}{ccccc}
-\frac{1}{T_{P_{i}}} & \frac{K_{p_{i}}}{T_{p_{i}}} & 0 & 0 & -\frac{K_{p_{i}}}{2 \pi T_{p_{i}}} \sum_{\substack{j=1 \\
j \neq i}}^{N} K_{s_{i j}} \\
0 & -\frac{1}{T_{T_{i}}} & \frac{1}{T_{T_{i}}} & 0 & 0 \\
-\frac{1}{R_{i} T_{P_{i}}} & 0 & -\frac{1}{T_{G_{i}}} & -\frac{1}{T_{G_{i}}} & 0 \\
K_{E_{i}} K_{B_{i}} & 0 & 0 & 0 & \frac{K_{E_{i}}}{2 \pi} \sum_{\substack{j=1 \\
j \neq i}}^{N} K_{s_{i j}} \\
2 \pi & 0 & 0 & 0 & 0
\end{array}\right] \text {, } \\
& \bar{H}_{i j}=\left[\begin{array}{ccccc}
0 & 0 & 0 & 0 & \frac{K_{P_{i}}}{2 \pi T_{P_{i}}} K_{s_{i j}} \\
0 & 0 & 0 & 0 & 0 \\
0 & 0 & 0 & 0 & 0 \\
0 & 0 & 0 & 0 & -\frac{K_{E_{i}}}{2 \pi} K_{s_{i j}} \\
0 & 0 & 0 & 0 & 0
\end{array}\right] \text {, } \\
& \bar{B}_{i}=\left[\begin{array}{lllll}
0 & 0 & \frac{1}{T_{G_{i}}} & 0 & 0
\end{array}\right]^{T}, \\
& \bar{F}_{i}=\left[\begin{array}{ccccc}
-\frac{K_{P_{i}}}{T_{P_{i}}} & 0 & 0 & 0 & 0
\end{array}\right]^{T} \text {, } \\
& x_{i}(t)=\left[\begin{array}{lll}
\Delta f_{i}(t) & \Delta P_{g_{i}}(t) & \Delta X_{g_{i}}(t) \Delta E_{i}(t) \Delta \delta_{i}(t)
\end{array}\right]^{T}
\end{aligned}
$$

and $x_{i}(t) \in R^{n_{i}}$ is the states of the $i$ th area subsystem, $x_{j}(t) \in R^{n_{j}}$ is the states of interconnected subsystems $j=$ $1,2, \ldots, N$ and $j \neq i, u_{i}(t) \in R^{m_{i}}$ is the control vector, and $\Delta P_{d_{i}}(t) \in R^{k_{i}}$ is the vector of load disturbance. Variables $\Delta f_{i}(t), \Delta P_{g_{i}}(t), \Delta X_{g_{i}}(t), \Delta E_{i}(t)$, and $\Delta \delta_{i}(t)$ are the changes of frequency, power output, governor valve position, integral control, and rotor angle deviation. $T_{G_{i}}, T_{t_{i}}$, and $T_{P_{i}}$ are the time constants of governor, turbine, and power system. $K_{P_{i}}, R_{i}, K_{E_{i}}$, and $K_{B_{i}}$ are power system gain, speed regulation coefficient, integral control gain, and frequency bias factor.
$K_{s_{i j}}$ is the interconnection gain between areas $i$ and $j(i \neq j)$. The dimensions of system matrices in (6) are $\bar{A}_{i} \in R^{n_{i} \times n_{i}}$, $\bar{H}_{i j} \in R^{n_{i} \times n_{i}}, \bar{B}_{i} \in R^{n_{i} \times m_{i}}, \bar{F}_{i} \in R^{n_{i} \times k_{i}}$. If there is no power exchange between $i$ and $j, K_{s i j}=0$.

Furthermore, since it is very difficult to find the exact values of the system parameters $\bar{A}_{i}, \bar{B}_{i}, \bar{H}_{i j}$, and $\bar{F}_{i}$ because of nonlinear and dynamic multi-area interconnected power system, the dynamic equation (6), the following general model can be drawn:

$$
\begin{aligned}
\dot{x}_{i}(t)= & {\left[A_{i}+\Delta A_{i}\left(x_{i}, t\right)\right] x_{i}(t) } \\
& +\left[B_{i}+\Delta B_{i}\left(x_{i}, t\right)\right] u_{i}(t) \\
& +\sum_{\substack{j=1 \\
j \neq i}}^{N}\left[H_{i j}+\Delta H_{i j}\left(x_{j}, t\right)\right] x_{j}(t)+\bar{F}_{i} \Delta P_{d i}(t) \\
= & A_{i} x_{i}(t)+B_{i} u_{i}(t)+\sum_{\substack{j=1 \\
j \neq i}}^{N} H_{i j} x_{j}(t)+L_{i}\left(x_{i}, t\right)
\end{aligned}
$$

where $A_{i}, B_{i}$, and $H_{i j}$ are the nominal values of $\bar{A}_{i}, \bar{B}_{i}$, and $\bar{H}_{i j}$; the unknown matrices $\Delta A_{i}\left(x_{i}, t\right), \Delta B_{i}\left(x_{i}, t\right)$, and $\Delta H_{i j}\left(x_{j}, t\right)$ denote the parameter uncertainties and the modeling errors; and $L_{i}\left(x_{i}, t\right)$ is called the aggregated uncertainties and defined as

$$
L_{i}\left(x_{i}, t\right)=\Delta A_{i} x_{i}(t)+\Delta B_{i} u_{i}(t)+\sum_{\substack{j=1 \\ j \neq i}}^{N} \Delta H_{i j} x_{j}(t)
$$

$$
+\bar{F}_{i} \Delta P_{d i}
$$

The aggregated uncertainties $L_{i}\left(x_{i}, t\right)$ are assumed to be bounded and satisfy the following condition:

$$
\left\|L_{i}\left(x_{i}, t\right)\right\|<\sum_{\alpha=0}^{\beta} \delta_{i \alpha}\left\|x_{i}(t)\right\|^{\alpha}, \quad i=1,2, \ldots, N
$$

where $\delta_{i \alpha}, i=1,2, \ldots, N$ and $\alpha=0,1,2, \ldots, \beta$, are unknown positive constants. The positive integer $\beta$ is determined by 
the designer in accordance with the knowledge about the order of the lumped uncertainty. For example, if the lumped uncertainty in $i$ th area contains a term such as $x_{i}^{3}(t)$, then one may choose $\beta=3$. In practice, the bounds of the system uncertainty are often unknown in advance. So adaptive tuning laws given in (20) and (21) are proposed to estimate $L_{i}\left(x_{i}, t\right)$.

Remark 1. The current sliding mode control approaches for load frequency control of the multi-area interconnected power system are achieved under assumption that the norm of the lumped uncertainty is bounded by a positive constant $[21,23]$. That is $\left\|L_{i}\left(x_{i}, t\right)\right\| \leq h_{i}$ where $h_{i}$ is a positive constant. This condition is quite restrictive.

Remark 2. In this approach, the aggregated uncertainties $L_{i}\left(x_{i}, t\right)$ are bounded by more general function with $\beta$ order of state variable $x_{i}(t)$. Notably, the knowledge of the upper bounds on the uncertainties is not a required prerequisite for designing the decentralised adaptive double integral sliding mode controller. Therefore, (10) is a positive function, and it is just an extension of the condition given in Remark 1 .

\section{Decentralised Adaptive Double Integral Sliding Mode Controller Design}

First, let error $e_{i}(t)=x_{i}(t)-\tilde{x}_{i}(t), i=1,2, \ldots N$ in which $\tilde{x}_{i}(t)$ is the desired value. Then, a traditional integral sliding surface is given as below:

$$
\sigma_{i}(t)=S_{i}\left[e_{i}(t)+\varepsilon_{i} \int_{0}^{t} e_{i}(\tau) d \tau\right]
$$

where $\varepsilon_{i}$ is positive constant; $S_{i} \in R^{m_{i} \times n_{i}}$ is any full rank matrix such that $S_{i} B_{i}$ is invertible. Then, by the time derivative of $\sigma_{i}(t)$ and using (8), we have

$$
\begin{gathered}
\dot{\sigma}_{i}(t)=S_{i}\left[\dot{e}_{i}(t)+\varepsilon_{i} e_{i}(t)\right]=S_{i}\left[-\dot{\tilde{x}}_{i}(t)+A_{i} x_{i}(t)\right. \\
\left.+B_{i} u_{i}+\sum_{\substack{j=1 \\
i \neq j}}^{N} H_{i j} x_{j}+L_{i}+\varepsilon_{i} e_{i}(t)\right]
\end{gathered}
$$

The stability of (12) is assured if the traditional integral sliding mode control effort $u_{i}^{C I S M C}(t)$ is given as [25]

$$
\begin{gathered}
u_{i}^{\text {CISMC }}(t)=-\left(S_{i} B_{i}\right)^{-1}\left[-S_{i} \dot{\tilde{x}}_{i}(t)+S_{i} A_{i} x_{i}(t)\right. \\
\left.+\sum_{\substack{j=1 \\
i \neq j}}^{N} S_{j} H_{j i} x_{i}+\varepsilon_{i} S_{i} e_{i}(t)+\tau_{i} s a t\left(\sigma_{i}(t)\right)\right]
\end{gathered}
$$

where $\tau_{i}$ is positive constant and

$$
\text { sat }\left(\sigma_{i}(t)\right)= \begin{cases}1, & \sigma_{i}(t)>1 \\ \sigma_{i}(t), & \left|\sigma_{i}(t)\right| \leq 1 \\ -1, & \sigma_{i}(t)<-1\end{cases}
$$

Because the aggregated uncertainties $L_{i}\left(x_{i}, t\right)$ of the multiarea interconnected power system are usually unknown in advance in load frequency control approaches, the required control parameter $\delta_{i \alpha}$ to keep the system state within the boundary layer is hard to choose. Thus, an adaptive gain tuning law is adopted in the proposed double integral sliding mode controller to estimate the unknown upper bound of the aggregated uncertainties $L_{i}\left(x_{i}, t\right)$ and to improve the steadystate control performance. The double integral sliding surface is given as below:

$$
\begin{aligned}
& \sigma_{i}(t) \\
& \quad=S_{i}\left[e_{i}(t)+\varepsilon_{i} \int_{0}^{t} e_{i}(\tau) d \tau+\widehat{\varepsilon}_{i} \int_{0}^{t} \int_{0}^{t} e_{i}(\tau) d \tau d \tau\right]
\end{aligned}
$$

where $\varepsilon_{i}$ and $\widehat{\varepsilon}_{i}$ are positive constants; $S_{i} \in R^{m_{i} \times n_{i}}$ is any full rank matrix such that $S_{i} B_{i}$ is invertible. By the time derivative of $\sigma_{i}(t)$ and using (8), we can achieve

$$
\begin{aligned}
& \dot{\sigma}_{i}(t)=S_{i}\left[-\dot{\tilde{x}}_{i}(t)+A_{i} x_{i}(t)+B_{i} u_{i}(t)+\sum_{\substack{j=1 \\
i \neq j}}^{N} H_{i j} x_{j}\right. \\
& \left.\quad+L_{i}\left(x_{i}, t\right)+\varepsilon_{i} e_{i}(t)+\widehat{\varepsilon}_{i} \int_{0}^{t} e_{i}(\tau) d \tau\right]
\end{aligned}
$$

In order to achieve the stability of the multi-area power system represented by (8), the decentralised adaptive double integral sliding mode control law is designed as below:

$$
u_{i}(t)=u_{i}^{r}(t)+u_{i}^{a d p}(t)+u_{i}^{s w}(t)
$$

where

$$
\begin{aligned}
& u_{i}^{r}(t)=-\left(S_{i} B_{i}\right)^{-1}\left[-S_{i} \dot{\tilde{x}}_{i}(t)+S_{i} A_{i} x_{i}(t)\right. \\
& \left.+\sum_{\substack{j=1 \\
i \neq j}}^{N} S_{j} H_{j i} x_{i}+\varepsilon_{i} S_{i} e_{i}(t)+\widehat{\varepsilon}_{i} S_{i} \int_{0}^{t} e_{i}(\tau) d \tau\right],
\end{aligned}
$$

and

$$
u_{i}^{s w}(t)=-\left(S_{i} B_{i}\right)^{-1} \tau_{i} s a t\left(\sigma_{i}(t)\right)
$$

and the proposed adaptive controller for tackling the system lumped uncertainty is designed as 


$$
u_{i}^{a d p}(t)=-\sum_{\alpha=0}^{\beta} \widehat{\delta}_{i \alpha}(t)\left(S_{i} B_{i}\right)^{-1}\left\|S_{i}\right\|\left\|x_{i}(t)\right\|^{\alpha}
$$

$$
i=1,2, \ldots, N
$$

where

$$
\dot{\hat{\delta}}_{i \alpha}(t)=\lambda_{i \alpha}\left\|S_{i}\right\|\left\|x_{i}(t)\right\|^{\alpha}, \quad i=1,2, \ldots, N
$$

in which $\lambda_{i \alpha}$ is the positive constant.

Remark 3. Equations (13), (17), and (18) show that the integral term $\int_{0}^{t} e_{i}(\tau) d \tau$ is only reflected in the proposed decentralised adaptive double integral sliding mode control law (17). Therefore, the control law (17) with I control feature results in improved steady-state error performance compared with the traditional integral sliding mode control (13).

Theorem 4. Considering the multi-area power system represented by (8), if the proposed decentralised adaptive double integral sliding mode controller given in (17) includes a robust controller $u_{i}^{r}(t)$ given in (18), an adaptive controller $u_{i}^{a d p}(t)$ given in (20) with adaptive gain tuning law (21), and a switching controller $u_{i}^{s w}(t)$ designed as (19), then the asymptotic stability of the multi-area power system is guaranteed.

Proof. Constructing a Lyapunov function

$$
V(t)=\sum_{i=1}^{N}\left[\left\|\sigma_{i}(t)\right\|+\sum_{\alpha=0}^{\beta} \frac{\widetilde{\delta}_{i \alpha}^{2}(t)}{2 \lambda_{i \alpha}}\right]
$$

where $\widetilde{\delta}_{i \alpha}(t)=\delta_{i \alpha}-\widehat{\delta}_{i \alpha}(t)$. The derivative of $V(t)$ is as

$$
\begin{aligned}
\dot{V}(t) & =\sum_{i=1}^{N}\left[\frac{\sigma_{i}^{T}(t)}{\left\|\sigma_{i}(t)\right\|} \dot{\sigma}_{i}(t)-\sum_{\alpha=0}^{\beta} \frac{\widetilde{\delta}_{i \alpha} \dot{\hat{\delta}}_{i \alpha}}{\lambda_{i \alpha}}\right] \\
& =\sum_{i=1}^{N} \frac{\sigma_{i}^{T}(t)}{\left\|\sigma_{i}(t)\right\|} S_{i}\left[\dot{e}_{i}(t)+\varepsilon_{i} e_{i}(t)+\widehat{\varepsilon}_{i} \int_{0}^{t} e_{i}(\tau) d \tau\right] \\
& -\sum_{i=1}^{N} \sum_{\alpha=0}^{\beta} \frac{\widetilde{\delta}_{i \alpha} \dot{\hat{\delta}}_{i \alpha}}{\lambda_{i \alpha}}=\sum_{i=1}^{N} \frac{\sigma_{i}^{T}(t)}{\left\|\sigma_{i}(t)\right\|} S_{i}\left[-\dot{\tilde{x}}_{i}(t)+\dot{x}_{i}(t)\right. \\
& \left.+\varepsilon_{i} e_{i}(t)+\widehat{\varepsilon}_{i} \int_{0}^{t} e_{i}(\tau) d \tau\right]-\sum_{i=1}^{N} \sum_{\alpha=0}^{\beta} \frac{\widetilde{\delta}_{i \alpha} \dot{\hat{\delta}}_{i \alpha}}{\lambda_{i \alpha}}
\end{aligned}
$$

Substituting (8) into (23) gives

$$
\begin{array}{r}
\dot{V}(t)=\sum_{i=1}^{N} \frac{\sigma_{i}^{T}(t)}{\left\|\sigma_{i}(t)\right\|} S_{i}\left[-\dot{\tilde{x}}_{i}(t)+A_{i} x_{i}(t)+B_{i} u_{i}(t)\right. \\
\left.+L_{i}\left(x_{i}, t\right)+\sum_{\substack{j=1 \\
i \neq j}}^{N} H_{i j} x_{j}+\varepsilon_{i} e_{i}(t)+\widehat{\varepsilon}_{i} \int_{0}^{t} e_{i}(\tau) d \tau\right]
\end{array}
$$

$$
\begin{aligned}
& -\sum_{i=1}^{N} \sum_{\alpha=0}^{\beta} \frac{\widetilde{\delta}_{i \alpha} \dot{\hat{\delta}}_{i \alpha}}{\lambda_{i \alpha}}=\sum_{i=1}^{N} \frac{\sigma_{i}^{T}(t)}{\left\|\sigma_{i}(t)\right\|}\left[-S_{i} \dot{\vec{x}}_{i}(t)\right. \\
& +S_{i} A_{i} x_{i}(t)+S_{i} B_{i} u_{i}(t)+S_{i} L_{i}+\sum_{\substack{j=1 \\
i \neq j}}^{N} S_{i} H_{i j} x_{j} \\
& \left.+\varepsilon_{i} S_{i} e_{i}(t)+\widehat{\varepsilon}_{i} \int_{0}^{t} S_{i} e_{i}(\tau) d \tau\right]-\sum_{i=1}^{N} \sum_{\alpha=0}^{\beta} \frac{\widetilde{\delta}_{i \alpha} \dot{\hat{\delta}}_{i \alpha}}{\lambda_{i \alpha}}
\end{aligned}
$$

Then, substituting (17)-(19) into (24), we achieve

$$
\begin{aligned}
\dot{V}(t) \leq & -\sum_{i=1}^{N} \tau_{i}\left\|\sigma_{i}\right\|-\sum_{i=1}^{N} \sum_{\alpha=0}^{\beta} \widehat{\delta}_{i \alpha}\left\|S_{i}\right\|\left\|x_{i}(t)\right\|^{\alpha} \\
& +\sum_{i=1}^{N} \sum_{\alpha=0}^{\beta} \delta_{i \alpha}\left\|S_{i}\right\|\left\|x_{i}(t)\right\|^{\alpha}-\sum_{i=1}^{N} \sum_{\alpha=0}^{\beta} \frac{\widetilde{\delta}_{i \alpha} \dot{\vec{\delta}}_{i \alpha}}{\lambda_{i \alpha}} \\
= & -\sum_{i=1}^{N} \tau_{i}\left\|\sigma_{i}\right\|-\sum_{i=1}^{N} \sum_{\alpha=0}^{\beta}\left(\delta_{i \alpha}-\widetilde{\delta}_{i \alpha}\right)\left\|S_{i}\right\|\left\|x_{i}(t)\right\|^{\alpha} \\
& +\sum_{i=1}^{N} \sum_{\alpha=0}^{\beta} \delta_{i \alpha}\left\|S_{i}\right\|\left\|x_{i}(t)\right\|^{\alpha} \\
& -\sum_{i=1}^{N} \sum_{\alpha=0}^{\beta} \frac{\widetilde{\delta}_{i \alpha} \lambda_{i \alpha}\left\|S_{i}\right\|\left\|x_{i}(t)\right\|^{\alpha}}{\lambda_{i \alpha}}=-\sum_{i=1}^{N} \tau_{i}\left\|\sigma_{i}\right\|
\end{aligned}
$$

It is clear that $\dot{V}(t) \leq 0$. This implies that

$$
V(t) \leq V(0)=\sum_{i=1}^{N}\left[\left\|\sigma_{i}(0)\right\|+\sum_{\alpha=0}^{\beta} \frac{\widetilde{\delta}_{\alpha}^{2}(0)}{2 \lambda_{i \alpha}}\right]
$$

and therefore $V(t), \sigma_{i}(t)$, and $\tilde{\delta}_{\alpha}^{2}(t)$ are bounded. In addition, by combining (22) and (25), it can be obtained that

$$
\begin{aligned}
\dot{V}(t) & \leq-\bar{\tau} V(t)+\sum_{i=1}^{N} \sum_{\alpha=0}^{\beta} \frac{\tau_{i} \widetilde{\delta}_{i \alpha}^{2}(t)}{2 \lambda_{i \alpha}} \\
& \leq-\bar{\tau} V(t)+\sum_{i=1}^{N} \sum_{\alpha=0}^{\beta} \frac{\tau_{i} v_{i \alpha}}{2 \lambda_{i \alpha}}
\end{aligned}
$$

where $\bar{\tau}=\min \left(\tau_{i}\right), i=1,2, \ldots, N$ and $v_{i \alpha}$ is the upper bound of $\widetilde{\delta}_{i \alpha}^{2}(t)$. Then, it yields

$$
\begin{aligned}
0 & \leq V(t) \leq e^{-\bar{\tau} t} V(0)+\left[\sum_{i=1}^{N} \sum_{\alpha=0}^{\beta} \frac{\tau_{i} \nu_{i \alpha}}{2 \lambda_{i \alpha}} \int_{0}^{t} e^{-\bar{\tau}(t-\kappa)} d \kappa\right. \\
& =e^{-\bar{\tau} t}\left[V(0)-\sum_{i=1}^{N} \sum_{\alpha=0}^{\beta} \frac{\tau_{i} v_{i \alpha}}{2 \lambda_{i \alpha} \bar{\tau}}\right]+\sum_{i=1}^{N} \sum_{\alpha=0}^{\beta} \frac{\tau_{i} \nu_{i \alpha}}{2 \lambda_{i \alpha} \bar{\tau}}
\end{aligned}
$$




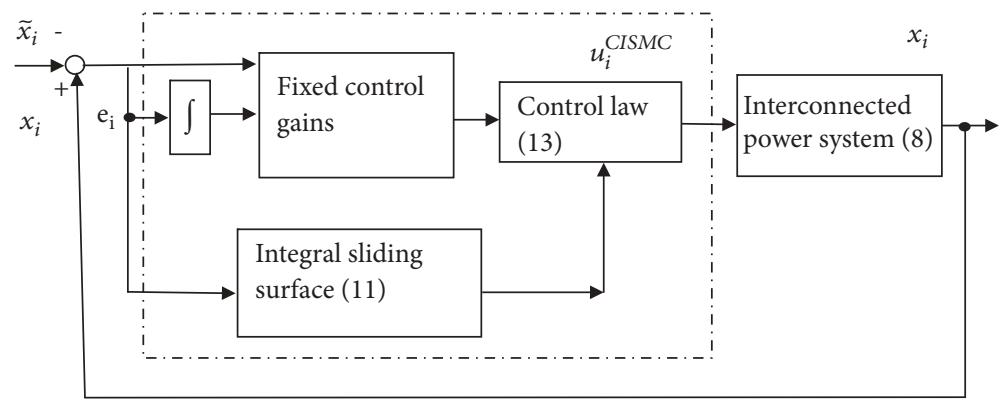

FIGURE 3: Configuration of traditional integral sliding mode control.

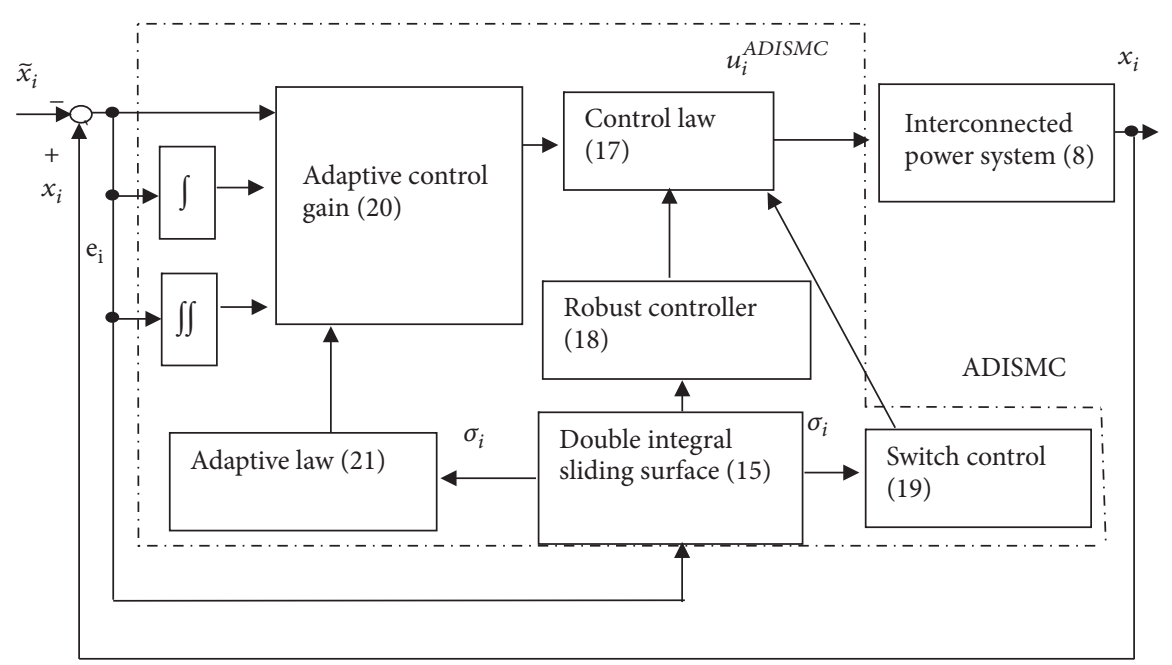

FIGURE 4: Configuration of the proposed decentralized adaptive double integral sliding mode control.

Using (28), the second derivative of $V(t)$ can be obtained such that

$$
\ddot{V}(t) \leq \bar{\tau}^{2} e^{-\bar{\tau} t}\left[V(0)-\sum_{i=1}^{N} \sum_{\alpha=0}^{\beta} \frac{\tau_{i} \nu_{i \alpha}}{2 \lambda_{i \alpha} \bar{\tau}}\right]
$$

Taking that the limit $t$ approaches infinity on both sides of (29), it can be shown that

$$
\begin{aligned}
\lim _{t \rightarrow \infty} \ddot{V}(t) & \leq \lim _{t \rightarrow \infty}\left\{\bar{\tau}^{2} e^{-\bar{\tau} t}\left[V(0)-\sum_{i=1}^{N} \sum_{\alpha=0}^{\beta} \frac{\tau_{i} \nu_{i \alpha}}{2 \lambda_{i \alpha} \bar{\tau}}\right]\right\} \\
& =0
\end{aligned}
$$

It is clear that $\ddot{V}(t)$ is also bounded. Hence, $\dot{V}(t)$ is uniformly continuous. It can be seen from (25), (26), and (30) that $V(t)$ is bounded, $\dot{V}(t)$ is negative semi-definite, and $\dot{V}(t)$ is uniformly continuous. By applying Barbalat's lemma (or the Lyapunovlike lemma) [25] to (25), (26), and (30) we achieve

$$
\lim _{t \longrightarrow \infty} \dot{V}(t)=0
$$

According to (25), we obtain

$$
\lim _{t \rightarrow \infty} \dot{V}(t)=\lim _{t \rightarrow \infty}\left[-\sum_{i=1}^{N} \tau_{i}\left\|\sigma_{i}\right\|\right]=0
$$

It can be seen that $\lim _{x \rightarrow \infty} \sigma_{i}(t)=0$. Thus, by standard linear control arguments, $\lim _{x \rightarrow \infty} e_{i}(t)=0$ is obtained and the asymptotic stability of the multi-area power system can be assured.

Remark 5. To clarify the differences and improvements of the control approaches including traditional integral sliding mode control and decentralised adaptive double integral sliding mode control, the block diagram of the above control approaches is given in Figures 3 and 4. First, the sliding mode control using traditional integral sliding surface is given in Figure 3. From (13) and Figure 3, the sliding mode control using traditional integral sliding surface results in $u_{i}^{C I S M C}(t)$ with fixed control gains. However, the bound of the aggregated uncertainties $L_{i}\left(x_{i}, t\right)$ of the multi-area interconnected power system is usually unknown in advance in load frequency control approaches. Thus, the required control parameter $\delta_{i \alpha}$ to keep the system state within the boundary layer is hard to choose. For this reason, an adaptive gain tuning law is adopted in the proposed double integral sliding mode controller to estimate the unknown upper bound of the system uncertainties $L_{i}\left(x_{i}, t\right)$ and to improve the steady-state control performance. The proposed decentralised adaptive double integral sliding mode control combines the merits of the integral sliding mode control 
TABle 1: Parameters of three-area interconnected power system [21].

\begin{tabular}{lcccccccc}
\hline Area & $T_{P i}$ & $K_{P i}$ & $T_{T i}$ & $T_{G i}$ & $R_{i}$ & $K_{E i}$ & $K_{B i}$ \\
\hline 1 & 20 & 120 & 0.3 & 0.08 & 2.4 & 10 & 0.41 & 0.55 \\
2 & 25 & 112.5 & 0.33 & 0.072 & 2.7 & 9 & 0.37 \\
3 & 20 & 115 & 0.35 & 0.07 & 2.5 & 7.1 & 0.65 \\
\hline
\end{tabular}

and adaptive control. Moreover, the proposed decentralised adaptive double integral sliding mode control law can be easily applied to the multi-area interconnected power systems with a general structure given in (8).

Remark 6. The adaptive control (20) with adaptation law (21) offers the advantage that no a priori knowledge about the bounds of $\widehat{\delta}_{i \alpha}$ is required as it adaptively estimates the bounds of $\widehat{\delta}_{i \alpha}$ and also ensures that the adaptive gain does not get overestimated. Therefore the adaptive control (20) with adaptation law (21) will reduce the saturated control effort.

Remark 7. In this approach, lumped uncertainties $L_{i}\left(x_{i}, t\right)$ are bounded by more general structure than the one in [21, 23]; the adaptive gain tuning law (20) and (21) is adopted to estimate the unknown upper bound of the aggregated uncertainties $L_{i}\left(x_{i}, t\right)$. In addition, the steady-state error of frequency deviation is alleviated by using the proposed decentralised double integral sliding mode control law (17). Therefore, the two major limitations in $[21,23]$ (the aggregated uncertainties are bounded by a positive constant and steady-state errors will be existent in the frequency deviation) are both eliminated.

\section{Application Results}

To test the effectiveness and superiority of proposed double integral sliding mode control approach, and to compare the results with the recent applied sliding mode control techniques [21], three-area interconnected power system networks are considered as the test system with its parameters given in [21] (see Table 1).

The parameters $\varepsilon_{i}$ and $\widehat{\varepsilon}_{i}$ in the proposed decentralised adaptive double integral sliding mode control law (17)-(18) can be reflected as the $\mathrm{P}$ control and I control gains $\mathrm{K}_{\mathrm{P}}$ and $K_{\mathrm{I}}$, respectively, and will affect the control performance significantly. If $\varepsilon_{i}$ is made large to get adequately small steady-state error, the damping may be much too low for satisfactory transient response. The integral gain $\widehat{\varepsilon}_{i}$ can be selected purely to provide an acceptable dynamic response; however, typically it will cause instability if raised sufficiently high. In addition, the constant $\lambda_{i \alpha}$ in (21) determines the convergence rate of the estimated bounds $\widehat{\delta}_{i \alpha}$. Practically, any constant $\lambda_{i \alpha}$ can be used to estimate the disturbance but a large value only is used for faster estimation of disturbance resulting in larger band of the bounded region and vice versa. Therefore, all the control parameters should be further adjusted manually considering the trade-off between control

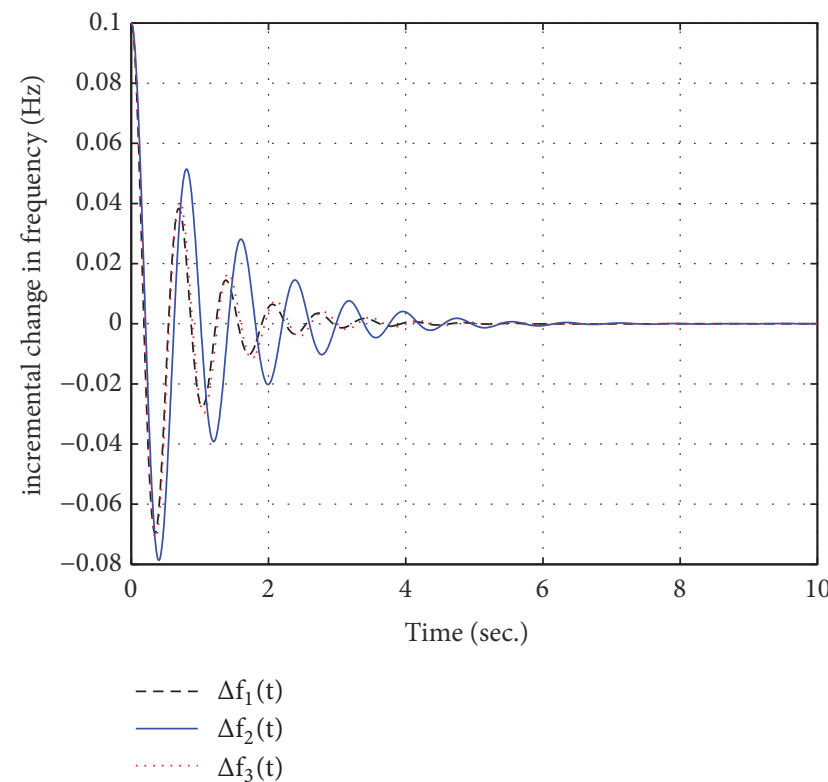

FIgURE 5: The frequency deviations (Hz) for Case 1: $\Delta f_{1}(t)$ (dashed), $\Delta f_{2}(t)$ (solid), $\Delta f_{3}(t)$ (dotted).

performance and system stability. As a result, the control parameters of the proposed decentralised adaptive double integral sliding mode control law are chosen as follows: $\varepsilon_{1}=$ $20.5, \varepsilon_{2}=20.5, \varepsilon_{3}=20.5, \widehat{\varepsilon}_{1}=20.5, \widehat{\varepsilon}_{2}=20.5, \widehat{\varepsilon}_{3}=20.5$, $\tau_{1}=0.1, \tau_{2}=0.1, \tau_{3}=0.1, \beta=2, \delta_{10}=\delta_{11}=\delta_{12}=1$, $\delta_{20}=\delta_{21}=\delta_{22}=1, \delta_{30}=\delta_{31}=1$. The sliding surface parameters are selected as $S_{1}=\left[\begin{array}{lllll}1 & 0.1 & 0.0065 & 2.5 & 0.4\end{array}\right], \mathrm{S}_{2}=$

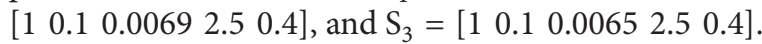

Case 1. In this base case, nominal parameters of the multiarea power system are used. No disturbances are assumed to be acting on the system; that is, $L_{i}\left(x_{i}, t\right)=0$.

Simulation results of the frequency deviations of the three-area interconnected power system for Case 1 using the proposed double integral sliding mode controller are shown in Figure 5. It is observed from Figure 4 that the frequency deviations converge to zero in about 6 seconds. Figure 6 shows that the tie line power deviation reaches zero with the proposed double integral sliding mode controller. In comparing the simulation results with the results given by [21], the proposed double integral sliding mode controller (17)-(21) can assure not only a fast response but also the smaller overshoot. 


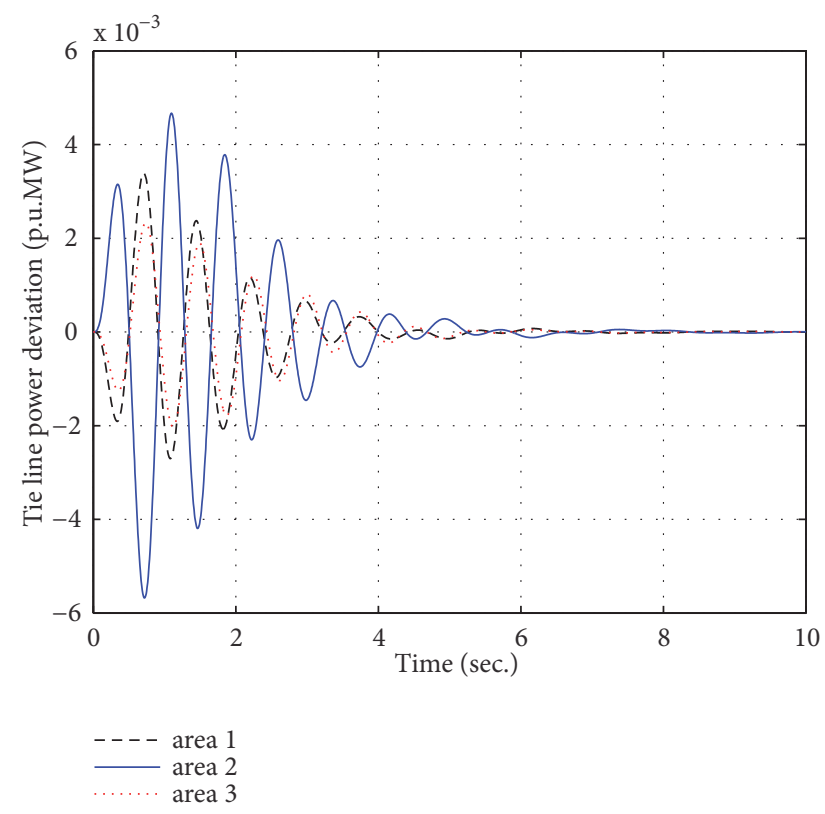

FIgURE 6: Tie line power deviation for Case 1.

Case 2. The main goal of designing a controller is its ability to work well under uncertain environment. In this case, the system performance with the proposed double integral sliding mode controller is test under matched parameter uncertainties and load disturbances.

Load disturbances of $\Delta P_{d_{1}}(t)=0.01 \mathrm{pu}, \Delta P_{d_{2}}(t)=0.015$ pu, and $\Delta P_{d_{3}}(t)=0.02$ pu are assumed to occur in areas 1,2 , and 3 , respectively. The matched parameter uncertainties in the three areas are given in [21]

$$
\begin{aligned}
& \Delta A_{1} \\
& =\left[\begin{array}{ccccc}
0 & 0 & 0 & 0 & 0 \\
0 & 0 & 0 & 0 & 0 \\
-2.26 \cos (t) & 2 \cos (t) & -2.604 \cos (t) & 3 \cos (t) & 0 \\
0 & 0 & 0 & 0 & 0 \\
0 & 0 & 0 & 0 & 0
\end{array}\right]
\end{aligned}
$$

and $\Delta A_{2}=\Delta A_{3}=\Delta A_{1}$.

The closed-loop responses for each control area using the proposed double integral sliding mode controller are shown in Figures 7 and 8. It is observed from Figures 7 and 8 that the system responses are better, in terms of overshoots and settling time, compared to the one proposed in [21].

Case 3. In the previous case, the proposed double integral sliding mode controller has its ability to work well under matched parameter uncertainties and load disturbances. However, in reality, there exists mismatched parameter uncertainties in the state matrix (due to wearing out of components or variation of operating points), mismatched interconnections among subsystems (due to unknown or variations in synchronizing power coefficients), and unknown disturbances. Therefore, the robustness against those aggregated

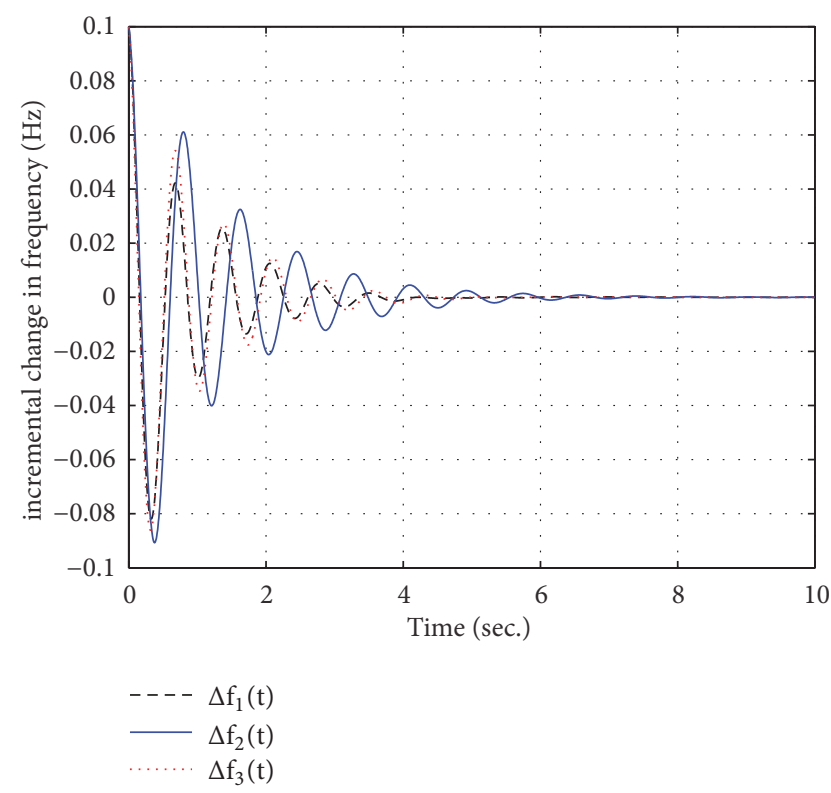

Figure 7: The frequency deviations $(\mathrm{Hz})$ for Case 2: $\Delta f_{1}(t)$ (dashed), $\Delta f_{2}(t)$ (solid), $\Delta f_{3}(t)$ (dotted).

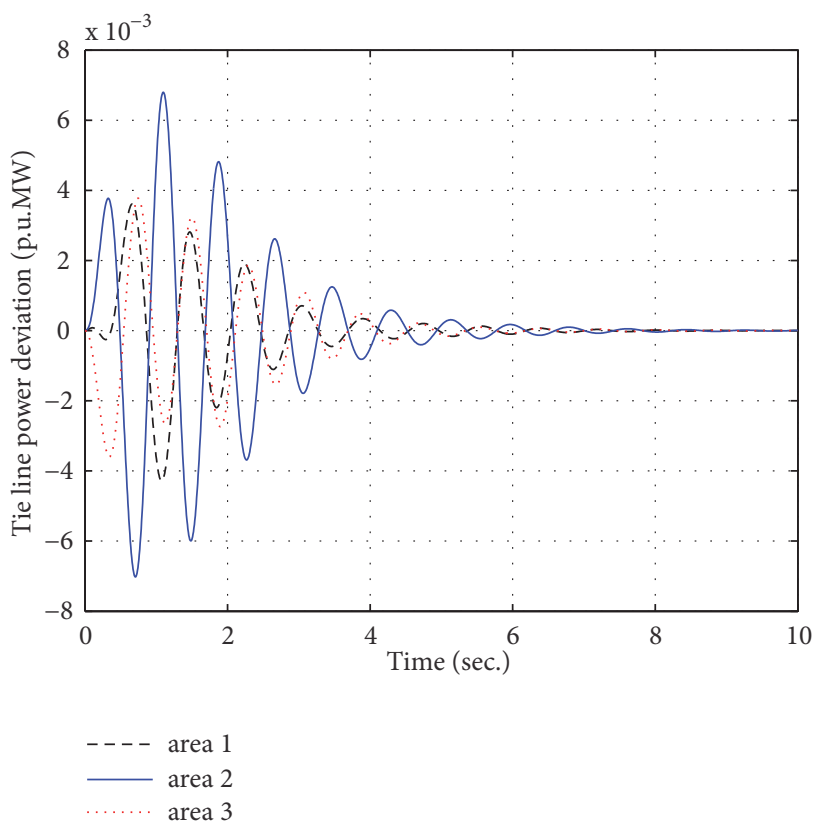

Figure 8: Tie line power deviation for Case 2.

uncertainties needs to be tested. The mismatched parameter uncertainties in the state matrix in three areas are chosen as follows:

$$
\Delta A_{1}=\left[\begin{array}{ccccc}
0 & \Delta f_{1} & 0 & 0 & 0 \\
\sin (t) & 0 & 0 & 0 & 0 \\
0 & 0 & \cos (t) & \cos (t) & 0 \\
0 & 0 & 0 & 0 & \cos (t) \\
\cos (t) & 0 & 0 & 0 & 0
\end{array}\right],
$$




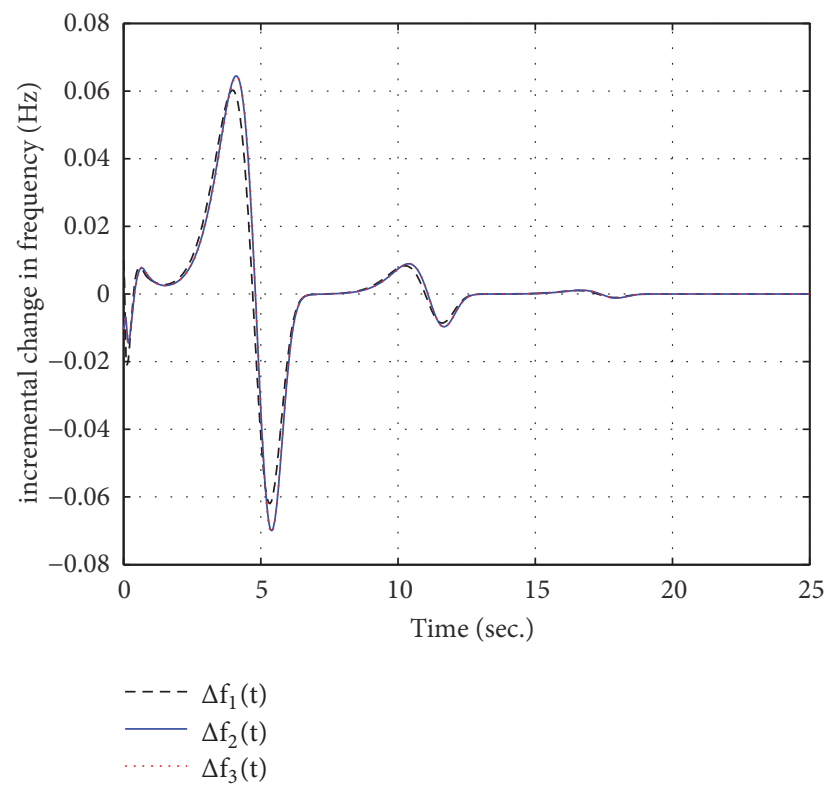

FIgure 9: The frequency deviations $(\mathrm{Hz})$ for Case 3: $\Delta f_{1}(t)$ (dashed), $\Delta f_{2}(t)$ (solid), $\Delta f_{3}(t)$ (dotted).

and $\Delta A_{2}=\Delta A_{3}=\Delta A_{1}$. The mismatched interconnections among subsystems are assumed as

$$
\Delta H_{12}=\left[\begin{array}{ccccc}
0 & 0 & 0 & 0 & 0.178 \cos (t) \\
0 & 0 & 0 & 0 & 0 \\
0 & 0 & 0 & 0 & 0 \\
0 & 0 & 0 & 0 & -0.296 \sin (t) \\
0 & 0 & 0 & 0 & 0
\end{array}\right]
$$

and $\Delta H_{23}=\Delta H_{31}=\Delta H_{12}$. The $\operatorname{rank}\left[B_{i}, \Delta A_{i}\right] \neq \operatorname{rank}\left[B_{i}\right]$ and $\operatorname{rank}\left[B_{i}, \Delta H_{i j}\right] \neq \operatorname{rank}\left[B_{i}\right]$ for $\mathrm{i}=1,2,3$; therefore parameter uncertainties in this case are mismatched uncertainties. The aggregated uncertainties of interconnected power systems include mismatched parameter uncertainties, nonlinear terms, and load disturbances. The load disturbances can be approximated by two parts, that is, nonfrequency-sensitive load change and frequency-sensitive load change. In this case, the load disturbance for the ith area subsystem $\Delta P_{d_{i}}(t)$ is taken to be the function of frequency deviation as $\Delta P_{d_{i}}(t)=$ $0.015+0.015 \Delta f_{i}(t)+0.015 \Delta f_{i}^{2}(t)$ for $\mathrm{i}=1,2,3$. Then, the aggregated uncertainties of the first area, the second area, and the third area are assumed to be bounded by $\left\|L_{1}\left(x_{1}, t\right)\right\| \leq$ $1+x_{1}+x_{1}^{2},\left\|L_{2}\left(x_{2}, t\right)\right\| \leq 1+x_{2}+x_{2}^{2}$, and $\left\|L_{3}\left(x_{3}, t\right)\right\| \leq 1+x_{3}+x_{3}^{2}$, respectively.

Figures 9 and 10 show the plots of the frequency deviation and the tie line power deviation for the three-area interconnected power system when the mismatched parameter uncertainties are used and input disturbances are assumed to be acting on the system. It is observed that the frequency deviation and the tie line power deviation converge to zero in about 15 seconds.

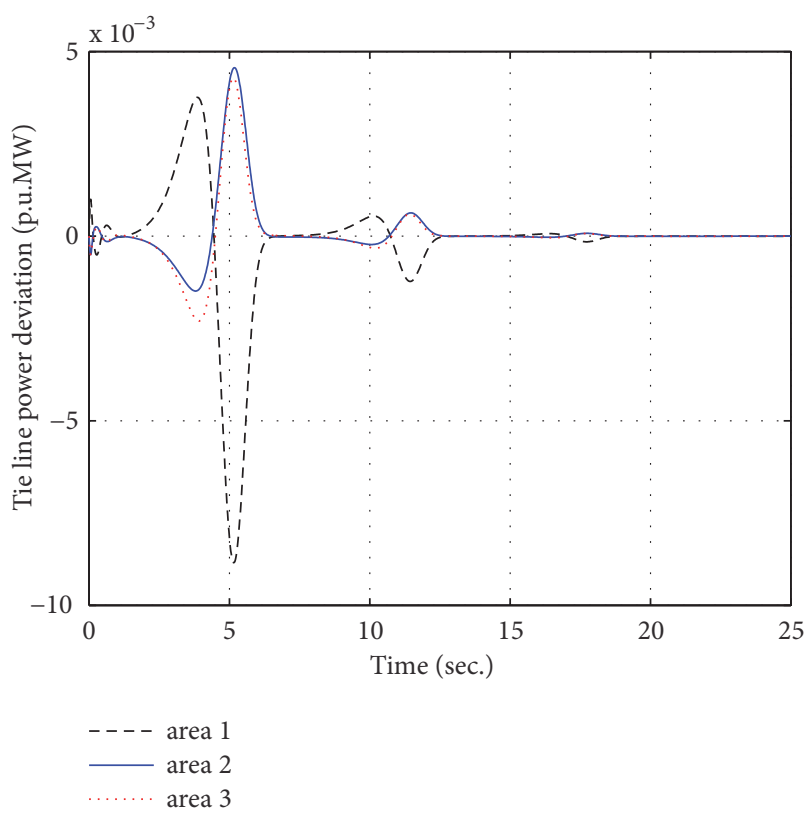

FIGURE 10: Tie line power deviation for Case 3.

Even under mismatched parameter uncertainties and load disturbances, the proposed double integral sliding mode controller successfully preserves system stability. Accordingly, the proposed design becomes a good choice to cope with mismatched parameter uncertainties in the state matrix, mismatched interconnections among subsystems, and load disturbances.

Remark 8. Comparing the simulation results for the three cases, the proposed double integral sliding mode controller is robust to disturbances acting on the system associated with variations of the matched and mismatched parameter uncertainties. Noticeably, the performance of the proposed double integral sliding mode controller can strongly outperforms that of the sliding mode controller proposed in [21].

Remark 9. In this simulation, the norm of lumped uncertainty of the three-area interconnected power system is bounded by unknown function of system states with the second order $\left(x_{1}^{2}, x_{1}^{2}\right.$, and $\left.x_{3}^{2}\right)$. Therefore, the method given in $[21,23]$ can not applied for this kind of lumped uncertainty.

\section{Conclusions}

This paper presents a new load frequency controller for multi-area power system where the aggregated uncertainties are bounded by unknown function of state variables, which is more general structure. An adaptive gain tuning law is adopted to estimate the unknown upper bound of the aggregated uncertainties. A double integral sliding surface based adaptive sliding mode controller is proposed to alleviate the steady-state errors and improve the transient performance of the closed loop system. Simulation results show that the proposed double integral sliding mode controller successfully 
preserves system stability and is robust to disturbances acting on the system associated with variations of the matched and mismatched parameter uncertainties. Moreover, comparing the simulation results with the conventional sliding mode control, the proposed decentralized adaptive double integral sliding mode controller can assure not only a fast response but also the smaller overshoot. Therefore, the proposed double integral sliding mode controller becomes a good choice to cope with matched and mismatched parameter uncertainties and load disturbances in multi-area interconnected power systems.

\section{Appendix}

\section{Barbalat's Lemma (or the Lyapunov-Like Lemma) [25]}

If a scalar function $V(t)$ satisfies the conditions that $V(t)$ is lower bounded, $\dot{V}(t)$ is negative semi-definite, and $\dot{V}(t)$ is uniformly continuous, then $\dot{V}(t) \longrightarrow 0$ as $t \longrightarrow \infty$ [25].

\section{Data Availability}

The data used to support the findings of this study are available from the corresponding author upon request.

\section{Conflicts of Interest}

The authors declare that they have no conflicts of interest.

\section{References}

[1] H. Bevrani, Y. Mitani, and K. Tsuji, "Robust decentralised loadfrequency control using an iterative linear matrix inequalities algorithm," IEE Proceedings Generation, Transmission and Distribution, vol. 151, no. 3, pp. 347-354, 2004.

[2] R. Thirunavukarasu and I. A. Chidambaram, "PI2 controller based coordinated control with Redox Flow Battery and Unified Power Flow Controller for improved Restoration Indices in a deregulated power system," Ain Shams Engineering Journal, vol. 7, no. 4, pp. 1011-1027, 2016.

[3] M. Farahani, S. Ganjefar, and M. Alizadeh, "PID controller adjustment using chaotic optimisation algorithm for multi-area load frequency control," IET Control Theory \& Applications, vol. 6, no. 13, pp. 1984-1992, 2012.

[4] S. Saxena and Y. V. Hote, "Decentralized PID load frequency control for perturbed multi-area power systems," International Journal of Electrical Power \& Energy Systems, vol. 81, pp. 405415, 2016.

[5] M. T. Alrifai, M. F. Hassan, and M. Zribi, "Decentralized load frequency controller for a multi-area interconnected power system," International Journal of Electrical Power \& Energy Systems, vol. 33, no. 2, pp. 198-209, 2011.

[6] H. Chen, R. Ye, X. Wang, and R. Lu, "Cooperative Control of Power System Load and Frequency by Using Differential Games," IEEE Transactions on Control Systems Technology, vol. 23, no. 3, pp. 882-897, 2015.

[7] F. Liu, Y. H. Song, J. Ma, S. Mei, and Q. Lu, "Optimal load-frequency control in restructured power systems," IEE
Proceedings Generation, Transmission and Distribution, vol. 150, no. 1, pp. 87-95, 2003.

[8] C. Zhao, U. Topcu, and S. H. Low, "Optimal load control via frequency measurement and neighborhood area communication," IEEE Transactions on Power Systems, vol. 28, no. 4, pp. 35763587, 2013.

[9] A. Abdennour, "Adaptive optimal gain scheduling for the load frequency control problem," Electric Power Components and Systems, vol. 30, no. 1, pp. 45-56, 2002.

[10] D. Rerkpreedapong, A. Hasanović, and A. Feliachi, "Robust load frequency control using genetic algorithms and linear matrix inequalities," IEEE Transactions on Power Systems, vol. 18, no. 2, pp. 855-861, 2003.

[11] F. Daneshfar and H. Bevrani, "Load-frequency control: a GAbased multi-agent reinforcement learning," IET Generation, Transmission \& Distribution, vol. 4, no. 1, pp. 13-26, 2010.

[12] S. Saxena and Y. V. Hote, "Load frequency control in power systems via internal model control scheme and model-order reduction," IEEE Transactions on Power Systems, vol. 28, no. 3, pp. 2749-2757, 2013.

[13] A. M. Ersdal, L. Imsland, and K. Uhlen, "Model Predictive Load-Frequency Control," IEEE Transactions on Power Systems, vol. 31, no. 1, pp. 777-785, 2016.

[14] C. L. R. Chang, Y. S. Wu, and J. S. Cheng, "Online estimation of system parameters for artificial intelligence applications to load frequency control. IET Generation," Transmission \& Distribution, vol. 5, pp. 895-902, 2010.

[15] F. Daneshfar, "Intelligent load-frequency control in a deregulated environment: continuous-valued input, extended classifier system approach," IET Generation, Transmission \& Distribution, vol. 7, no. 6, pp. 551-559, 2013.

[16] H. A. Yousef, K. Al-Kharusi, M. H. Albadi, and N. Hosseinzadeh, "Load frequency control of a multi-area power system: An adaptive fuzzy logic approach," IEEE Transactions on Power Systems, vol. 29, no. 4, pp. 1822-1830, 2014.

[17] H. Yousef, "Adaptive fuzzy logic load frequency control of multi-area power system," International Journal of Electrical Power \& Energy Systems, vol. 68, pp. 384-395, 2015.

[18] F. Liu, Y. Li, Y. Cao, J. She, and M. Wu, "A two-layer active disturbance rejection controller design for load frequency control of interconnected power system," IEEE Transactions on Power Systems, vol. 31, no. 4, pp. 3320-3321, 2016.

[19] Y. Liu, Q. Lan, C. Qian, W. Qian, and H. Chu, "Universal finiteTime observer design and adaptive frequency regulation of hydraulic turbine systems," IET Control Theory \& Applications, vol. 10, no. 4, pp. 363-370, 2016.

[20] K. Vrdoljak, N. Perić, and I. Petrović, "Sliding mode based loadfrequency control in power systems," Electric Power Systems Research, vol. 80, no. 5, pp. 514-527, 2010.

[21] Y. Mi, Y. Fu, C. Wang, and P. Wang, "Decentralized sliding mode load frequency control for multi-area power systems," IEEE Transactions on Power Systems, vol. 28, no. 4, pp. 43014309, 2013.

[22] D. Qian, S. Tong, and X. Liu, "Load frequency control for micro hydro power plants by sliding mode and model order reduction," Automatika - Journal for Control, Measurement, Electronics, Computing and Communications, vol. 56, no. 3, pp. 318-330, 2015.

[23] D. Qian, S. Tong, H. Liu, and X. Liu, "Load frequency control by neural-network-based integral sliding mode for nonlinear power systems with wind turbines," Neurocomputing, vol. 173, pp. $875-885,2016$. 
[24] Y. Mi, Y. Fu, D. Li, C. Wang, P. C. Loh, and P. Wang, "The sliding mode load frequency control for hybrid power system based on disturbance observer," International Journal of Electrical Power \& Energy Systems, vol. 74, pp. 446-452, 2016.

[25] J. J. E. Slotine and W. Li, Applied nonlinear control, Prentice-Hall, New York, NY., USA, 1991. 


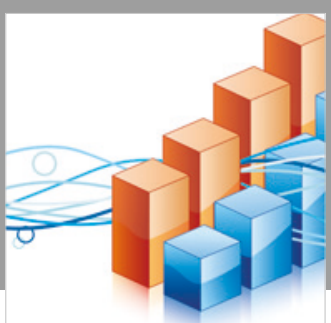

Advances in

Operations Research

\section{-n-m}
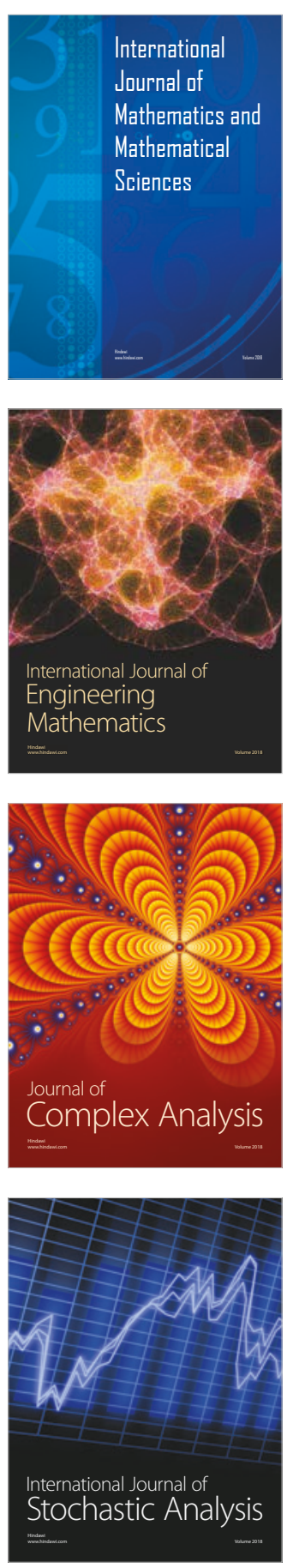
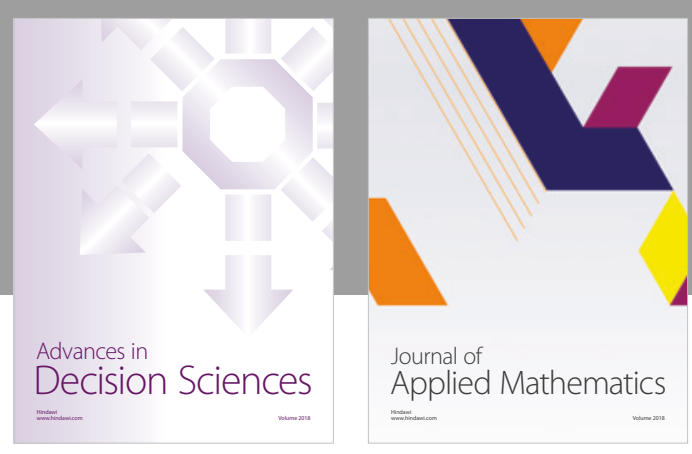

Journal of

Applied Mathematics
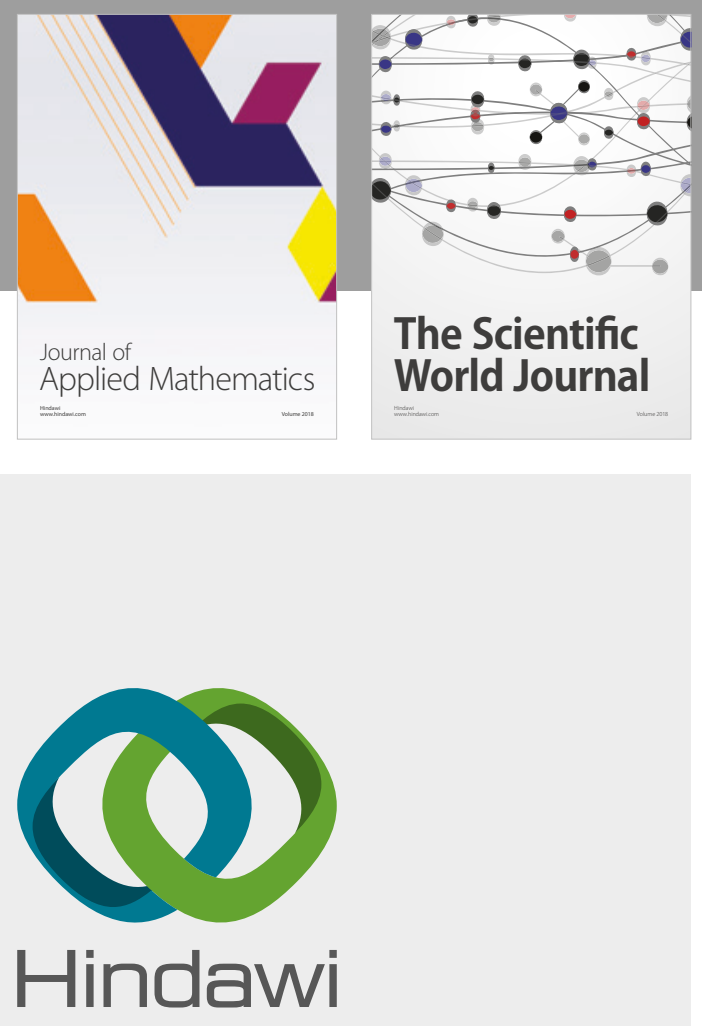

Submit your manuscripts at

www.hindawi.com

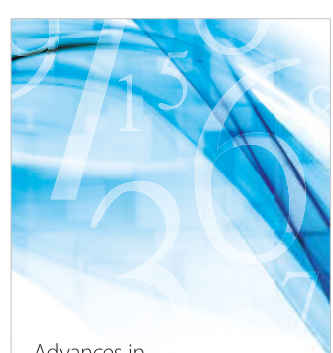

Advances in
Numerical Analysis
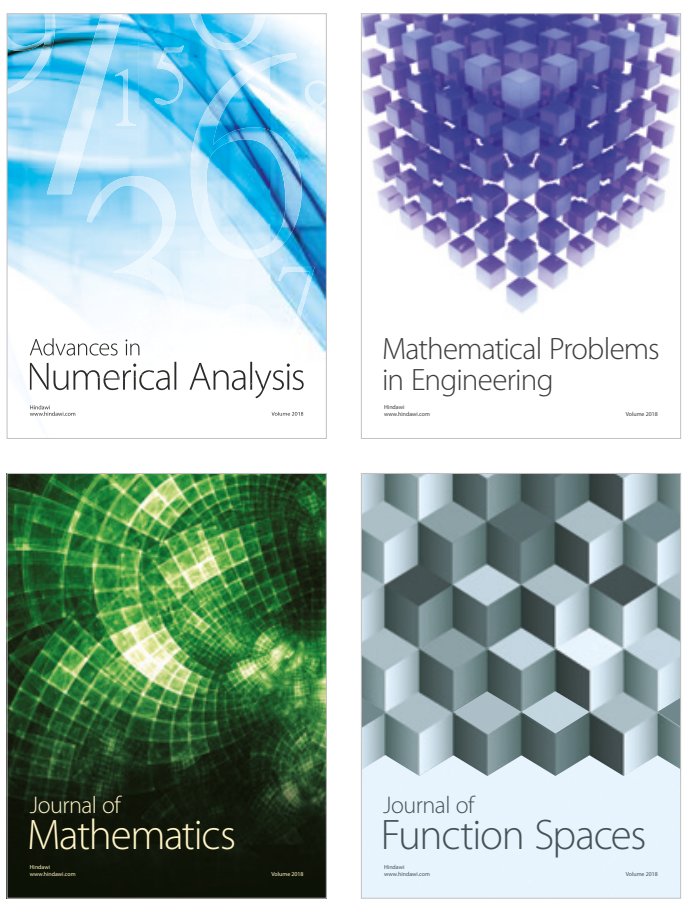

Mathematical Problems in Engineering

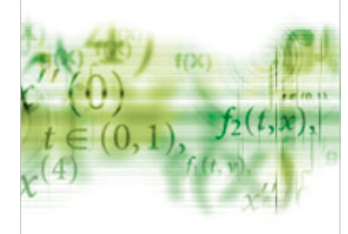

International Journal of

Differential Equations

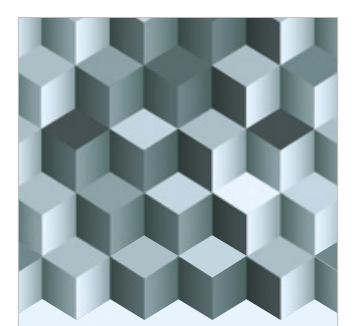

Journal of

Function Spaces

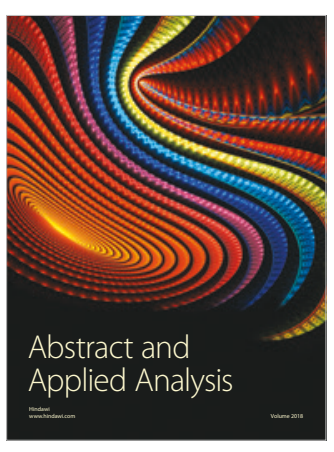

The Scientific

World Journal

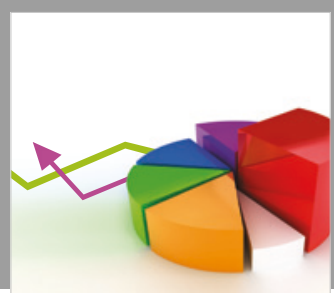

Journal of

Probability and Statistics
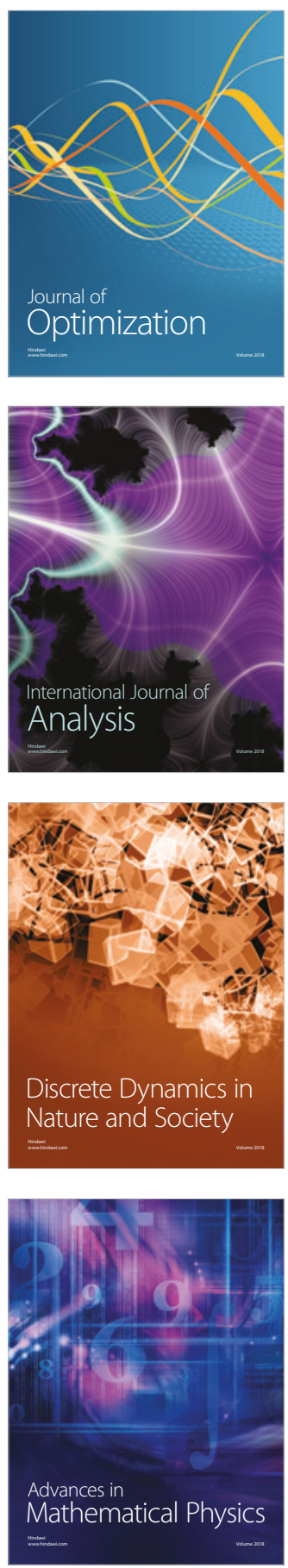